\title{
Fatores relacionados com a prática da episiotomia no Brasil: revisão de literatura
}

\section{Rafael Cleison Silva dos Santos ${ }^{1}$ e Rodrigo Gomes dos Santos ${ }^{2}$}

\begin{abstract}
1 Doutor em Ciências (Cuidado de Saúde) pela Universidade de São Paulo. Enfermeiro. Professor Adjunto do Departamento de Ciências Biológicas e da Saúde da Universidade Federal do Amapá, Brasil. E-mail: rcleison@unifap.br

2 Graduado em Licenciatura e Bacharelado em Enfermagem pela Universidade Federal do Amapá, Brasil. E-mail: r0drigog0mes@hot mail.com.br
\end{abstract}

RESUMO. Este trabalho teve como finalidade apresentar os fatores relacionados com a prática da episiotomia, no Brasil, estudados na literatura. Realizou-se uma revisão, mediante a seleção de artigos na Biblioteca Virtual de Saúde e a amostra final foi composta por oito estudos primários. Os fatores relacionados com a prática da episiotomia mais analisados pelos estudos brasileiros foram: condições maternas (idade, escolaridade, paridade, episiotomia anterior, rigidez perineal, altura do períneo); condições fetais (escore de Apgar, peso ao nascer, idade gestacional); condições assistenciais (posição materna, administração de ocitocina e misoprostol, manobra de Kristeller, formação do profissional e turno de trabalho).

Palavras-chave: Episiotomia. Parto. Períneo.

\section{Factors related to with the practice of episiotomy in Brazil: review}

ABSTRACT. The purpose of this study was to present the factors related to the practice of episiotomy in Brazil reported by the literature. It was conducted a review, by selecting the papers in the Virtual Health Library and the final sample was composed of eight primary studies. The factors related to the practice of episiotomy more analyzed by Brazilian studies were: maternal conditions (age, educational level, parity, previous episiotomy, perineal rigidity, perineum height); fetal conditions (Apgar score, birth weight, gestational age); birth care (maternal position, administration of oxytocin and misoprostol, fundus pressure maneuver, professional training and work shift).

Keywords: Episiotomy. Delivery. Perineum.

\section{INTRODUÇÃO}

A episiotomia é a ampliação cirúrgica do orifício vaginal, realizada no períneo durante o período expulsivo do parto, com a finalidade de impedir ou diminuir o trauma dos tecidos do canal do parto no desprendimento da cabeça fetal e de evitar lesões do polo cefálico devido à pressão sofrida de encontro ao períneo. Este procedimento é feito com tesoura ou bisturi e exige reparação por sutura (GHARAM, 1997).

Embora não seja conhecida exatamente quando a prática da episiotomia começou, um relatório, em 1741, sugeriu a abertura cirúrgica do períneo como meio de salvar a vida de uma criança e evitar lacerações graves perineais (OULD, 1741). A partir de então, tornou-se um dos procedimentos cirúrgicos mais realizados no mundo, mas sem 
evidências científicas de sua eficácia.

$\mathrm{Na}$ atualidade, as taxas de episiotomia seguem altas em todo o mundo e variam de 9,7\% (Norte da Europa - Suécia) a 96,2\% (América do Sul - Equador), com taxas mais baixas em países de língua inglesa (América do Norte - Canadá: 23,8\% e Estados Unidos: $32,7 \%$ ) e em muitos países (América do Sul Brasil: 94,2\%, África do Sul: 63,3\% e Ásia China: $82 \%$ ), permanecem elevadas, em três técnicas: mediana (também conhecida como perineotomia), lateral e médiolateral, sendo a perineotomia mais utilizada na América do Norte, enquanto a médiolateral é amplamente empregada na Europa e América Latina (BASTON; HALL, 2011).

Por isso, uma revisão Cochrane (CARROLLI; MIGNINI, 2012) foi realizada para avaliar os efeitos do uso da episiotomia restritiva em comparação com a de rotina durante o parto vaginal, e concluiu que o uso restritivo parece ter uma série de benefícios em comparação com a prática rotineira, tais como: menor trauma perineal posterior, menor necessidade de sutura e menor complicações, mas houve um aumento no risco de trauma perineal anterior espontâneo.

Os traumas perineais espontâneos podem ser únicos ou múltiplos, incluir a pele perineal ou estender-se aos músculos e ao esfíncter anal e ao ânus, e são classificados de acordo com os tecidos lesados, em virtude da intensidade e gravidade, as quais são aceitas internacionalmente: a) Períneo íntegro: não se observam rompimentos além das lacerações vulvares; b) Primeiro grau: rompimento que afeta a pele e as mucosas da vulva e da vagina; c) Segundo grau: lesões que, além das citadas como de primeiro grau, se estendem a músculos e fáscias do assoalho perineal; d) Terceiro grau: o rompimento que, junto aos músculos e fáscia, afeta o esfíncter anal externo e interno, sem chegar a provocar uma solução completa de sua continuidade (rompimento parcial); Quarto grau: é o rompimento mais abrangente e inclui às lesões de terceiro grau as que se estendem até a mucosa retal (KETTLE, 2005).

Para esse autor, os fatores associados a prática da episiotomia podem ser divididos em: a) Condições maternas: idade, etnia, paridade, cicatriz anterior e altura do períneo; b) Condições fetais: peso, perímetro cefálico, apresentação e variedade de posição fetal; c) Condições assistenciais: posição materna, duração expulsivo, uso de ocitocina, puxos dirigidos, uso de hialuronidase e proteção perineal.

Quanto à episiotomia de rotina, as justificativas do seu emprego são: prevenção do trauma perineal grave e das lesões desnecessárias do pólo cefálico do RN, relaxamento do assoalho pélvico, prolapso, retocele, cistocele, incontinência urinária, meIhoria da futura função sexual, o reparo torna-se facilitado por evitar lacerações irregulares e acelera o trabalho de parto (CUMIN; FARIA; SOLER, 2007).

No entanto, Costa et al. (2011) destacam que essa prática favorece inúmeras complicações como: extensão da lesão perineal, hemorragia, edema, infecção, hematomas, dispareunia, fistulas retovaginais, endometriose na cicatriz, disfunção sexual, aumento do índice de infecção, além de lesão do tecido muscular, nervoso, vasos, mucosa e pele.

Amorin e Katz (2008) evidenciaram em revisão de literatura que, o emprego da episiotomia de rotina não reduz a incidência de asfixia nos partos com ou sem episiotomia; não protege o assoalho pélvico materno, 
não previne incontinência urinária ou fecal e prolapso genital; a perda sanguínea é mais volumosa; o procedimento comportase como uma laceração de segundo grau e quando não realizada pode ocorrer ou não laceração ou surgir lacerações anteriores, de primeiro ou segundo grau, mas de bom prognóstico; aumenta a chance de dor pósparto e dispareunia; além de acarretar custos hospitalares mais altos.

Cumin, Faria e Soler (2007) reiteram que, relacionado ao feto, a episiotomia de rotina não previne lesões no pólo cefálico fetal, não melhora os escores de Apgar, não favorece o parto no caso de distocia de ombros (a distocia é óssea e não de desproporção com o períneo materno), além de não apresentar benefícios no parto de prematuros.

Nesse sentido, a Organização Mundial da Saúde (OMS), em seu "Guia prático para assistência ao parto normal" classifica as práticas relacionadas ao parto normal em quatro categorias: Categoria $\mathrm{A}$ : práticas demonstradamente úteis e que devem ser estimuladas; Categoria B: práticas claramente prejudiciais ou ineficazes e que devem ser eliminadas; Categoria C: práticas em relação às quais não existem evidências suficientes para apoiar uma recomendação clara e que devem ser utilizadas com cautela, até que mais pesquisas esclareçam a questão; Categoria D: práticas frequentemente utilizadas de modo inadequado. Nesse contexto, a episiotomia é considerada, nestes termos, como prática da categoria $\mathrm{D}$ : práticas frequentemente utilizadas de modo inadequado, o que contraria a indicação da episiotomia de rotina. A OMS recomenda o uso restrito da técnica, em apenas $10 \%$ dos partos normais, sendo suas indicações: sinais de sofrimento fetal, progressão insuficiente do parto e ameaça de lacera- ções de 3 o grau, incluindo laceração de 3 을 grau em parto anterior (OMS, 1996).

Este trabalho teve como finalidade apresentar os fatores relacionados com a prática da episiotomia no Brasil estudados na literatura.

\section{MÉTODO}

Trata-se de uma revisão de literatura, conforme proposto por Guanilo, Takahashi e Bertolozzi (2011).

A pergunta de revisão "Quais os fatores relacionados com a prática da episiotomia?" foi elaborada a partir da estratégia PICO (acrônimo utilizado para selecionar P: problem; I: intervention; C: comparison; e O: outcome), com a seguinte formulação: $P=$ parto; $I$ = episiotomia; $C$ = "indicação terapêutica"; $\mathrm{O}=$ "fatores predisponentes".

Para identificar os artigos acerca do assunto, realizou-se busca da literatura nacional no Portal da Biblioteca Virtual de Saúde (BVS), no período de agosto a outubro de 2014. Contudo, inicialmente, foi consultado o vocabulário estruturado de Descritores em Ciência da Saúde (DeCS); em seguida, utilizaram-se os seguintes descritores e operadores de busca: parto OR episiotomia OR "indicação terapêutica" OR "fatores predisponentes".

Para o refinamento dos artigos, foram empregados os seguintes critérios de inclusão: a) estudos observacionais; b) artigos cujos desfechos dos estudos eram os fatores predisponentes ou critérios mais utilizados para a prática da episiotomia; c) estudos identificados por meio de pergunta auto-aplicada ou aplicada pelo entrevistador; d) artigos em que os objetivos incluíssem a frequência ou prevalência dos desfechos, os fatores relacionados ou os critérios utiliza- 
dos à prática da episiotomia; e) estudos realizados com população alvo de parturientes, independentemente da paridade, em serviços de saúde do território brasileiro e publicados em periódicos na língua inglesa, portuguesa ou espanhola, entre os anos de 2004 a 2014; f) artigos com textos disponíveis na íntegra e com metodologia claramente descrita. Foram excluídos estudos cujo desfecho era apenas o trauma perineal espontâneo.

A extração dos dados dos artigos foi realizada através de instrumento contendo as seguintes informações: autores, ano de publicação, objetivos, métodos (local da pesquisa, desenho do estudo, população-alvo, forma de avaliação do desfecho), prevalência do desfecho e fatores associados.

A análise dos estudos encontrados foi feita de forma descritiva, em três etapas: primeiramente, fez-se leitura geral de todos os títulos dos artigos resultantes da busca, para estabelecer quais seriam incluídos; em seguida, processou-se a leitura seletiva dos resumos com a finalidade de determinar os textos que, de fato, interessariam à pesquisa; por último, realizou-se a leitura interpretativa dos artigos na íntegra, para relacionar os achados relacionados com a pergunta de pesquisa desta revisão. Finalmente, sintetizaram-se os resultados encontrados nos artigos selecionados. Além disso, foram mantidas a autenticidades das ideias, os conceitos e as definições dos autores.

\section{RESULTADO E DISCUSSÃO}

$\mathrm{Na}$ busca realizada, encontraram-se 448 artigos com texto completo. Após a aplicação dos filtros: texto na íntegra, assunto principal (episiotomia e períneo), idioma (inglês, português e espanhol), ano de pu- blicação (2004 a 2014) e tipo de documento (artigo), inicialmente, 186 artigos foram considerados elegíveis para o estudo. Pela leitura do título, foram selecionados 52 para a leitura dos resumos. Destes, 11 foram lidos na íntegra e oito estudos compuseram a amostra final.

Após a análise dos artigos (MELO JÚNIOR; LIMA; FREIRE, 2006; MOUTA et al., 2008; COSTA; SOUZA, 2009; CARVALHO; SOUZA; MORAES FILHO, 2010) observou-se que, no Brasil, a taxa de episiotomia é elevada, ultrapassando a recomendação da OMS, que é em torno de $10 \%$. No entanto, hospitais em que são implementados programas de educação em saúde apresentam redução nas taxas desse procedimento.

É consenso por parte dos autores referidos no parágrafo anterior que, apesar da prevalência da episiotomia ter sido superior à recomendada pela OMS, esta mostrou-se bem inferior à média nacional, que segundo Leal et al. (2014) é de 56,1\%.

Os fatores relacionados com a prática da episiotomia mais analisados pelos estudos brasileiros foram: condições maternas, condições fetais e condições assistenciais, que serão descritos a seguir.

\subsection{Condições maternas}

Os fatores maternos mais analisados pelos estudos foram: idade, paridade, episiotomia anterior, rigidez perineal, altura do períneo e escolaridade. Em estudo feito por Carvalho, Souza e Moraes Filho (2010), foram analisadas as seguintes condições maternas: idade, paridade, parto vaginal anterior e doenças associadas ao parto. Esse estudo verificou que a idade das mulheres submetidas à episiotomia variou entre $13 \mathrm{e}$ 43 anos. Os extremos da idade materna 
foram também associados, sendo a maioria adolescente, demonstrando um risco maior de realizar o procedimento. A primiparidade, a ausência de partos anteriores e as doenças presentes no momento de parto também são fatores relacionados à realização dessa técnica, sendo que as síndromes hipertensivas demonstraram maior prevalência dentre os fatores associados. Os autores sugerem que a indicação rotineira de episiotomia pode ainda estar influenciando na sua realização.

Outros autores que estudaram os fatores maternos relacionados à episiotomia foram Costa e Souza (2009), que analisaram a idade materna, a paridade e a procedência de mulheres. Nesse estudo foi verificada a associação quanto à idade materna e a paridade com a realização da episiotomia, ou seja, quanto maior o número de partos vaginais anteriores, menor a utilização da episiotomia.

As parturientes nulíparas e menores de 18 anos apresentaram tiveram maior prevalência de episiotomia. Os autores associam a esse resultado à indicação rotineira em nulíparas, considerando ainda que estas são mais jovens e com menor paridade. Outra indicação seria a prevenção de traumas perineais, no entanto, faltam estudos que evidenciem a ocorrência dos mesmos em muIheres jovens.

No estudo de Melo Júnior, Lima e Freire (2006), foi observado que as adolescentes e primíparas apresentaram um risco significativamente maior de serem submetidas à episiotomia. Foi ainda pesquisada a possível associação entre a procedência da mulher e o procedimento, porém esta foi insignificante.

Constatou-se, no estudo de Oliveira e Miquilini (2005), que a episiotomia foi pra- ticada na maioria dos partos de primigestas, tendo como justificativa principal a prevenção de laceração perineal. Esses autores, no entanto, relatam diminuição da necessidade do procedimento em um hospital-escola, com a simples adoção da deambulação livre durante o trabalho de parto. As condições maternas encontradas nesse estudo para indicar a episiotomia foram: rigidez perineal, primiparidade, períneo íntegro, episiotomia anterior, períneo curto e iminência de rotura, sendo a rigidez perineal e a primiparidade os critérios mais citados.

Na pesquisa de Riesco et al. (2011), a episiotomia foi mais frequente em mulheres de 15 a 25 anos e em mulheres nulíparas e que tiveram um parto vaginal anterior. Esses autores verificaram que quanto maior o número de partos, menor é a chance de ocorrer episiotomia, sendo que as mulheres sem parto vaginal anterior tem três vezes mais chance de serem submetidas a esse procedimento.

Santos et al. (2008) pesquisaram a frequência de lesões perineais ocorridas no parto vaginal, com ênfase na prática da episiotomia. As mulheres com idade entre $17 \mathrm{e}$ 26 anos foram as mais submetidas, sendo a média de idade de 24 anos. A maioria das mulheres estudou menos de oito anos e os autores sugerem que a baixa escolaridade torna a mulher mais vulnerável a intervenções obstétricas no parto, sem questionar sua realização. As primíparas, mais uma vez, tiveram maior indicação para o procedimento.

Scarabotto e Riesco (2006), por sua vez, pesquisaram os fatores relacionados ao trauma perineal no parto normal, a fim de verificarem a consistência das indicações da episiotomia quanto à prevenção de lacerações. Neste sentido, os autores constata- 
ram que o uso de episiotomia associada à idade materna não é justificável, uma vez que faltam evidências científicas para associar o trauma perineal à idade materna. A altura do períneo também não demonstrou associação significante com a ocorrência de lacerações perineais. Vale ressaltar que esse estudo abordou mulheres nulíparas, gestação a termo, com feto vivo em apresentação cefálica fletida, sem infusão endovenosa de ocitocina, sem preparo do períneo durante a gestação e parto na posição lateral esquerda.

Santos et al. (2008) e Oliveira e Miquilini (2005) fazem uma interessante observação em seus estudos, sobre a história obstétrica materna a respeito da indicação da episiotomia, sendo esta indicada para a maioria das primíparas e multíparas com episiotomia anterior, tornando-se assim, uma indicação universal na maioria dos partos, o que dificulta a análise de outros fatores.

\subsection{Condições fetais}

Os artigos selecionados nessa revisão abordaram as seguintes condições fetais relacionadas à episiotomia: escore de Apgar, peso ao nascer e idade gestacional. 0 estudo de Carvalho, Souza e Moraes Filho (2010) constatou que o uso da episiotomia não foi associado a sinais de sofrimento fetal durante o parto e às condições de nascimento do recém-nascido (Apgar e peso), sem significância estatística para justificar o procedimento como benefício para o feto.

Outros autores que encontraram resultados semelhantes foram Costa e Souza (2009), que averiguaram que a macrossomia não foi o único critério indicativo da episiotomia, sendo verificada a adoção do procedimento de forma semelhante nas diversas categorias de peso. Os autores ainda observaram associação significativa entre o escore de Apgar e a idade gestacional com a episiotomia, porém, com baixo grau de contingência, demonstrando pouco valor para o estudo.

Melo Júnior, Lima e Freire (2006) pesquisaram a associação entre episiotomia e peso ao nascer, idade gestacional e escore de Apgar e, assim como em outros estudos, não foi identificada associação significativa. Os autores constataram que as faixas de peso ao nascer mais citadas para o procedimento tiveram uma menor frequência de episiotomia.

No estudo de Mouta et al. (2008) foi associada a vitalidade do recém-nascido com a posição materna adotada durante o parto (horizontal ou vertical), sem associação com a episiotomia ou com a frequência de traumas perineais.

Em estudo realizado com médicos e enfermeiros obstetras em hospital universitário, com a finalidade de identificar os critérios adotados para a indicação da episiotomia, Oliveira e Miquilini (2005) contrasta com os outros estudos em relação à relevância do uso da episiotomia nas condições fetais de prematuridade, já que é indicada como procedimento que encurta o período expulsivo, evitando, desse modo, a compressão da cabeça fetal durante o trajeto pelo canal do parto; nesse estudo, também a macrossomia fetal que foi responsável pela maioria das mulheres submetidas a episiotomia.

Em outro trabalho, Riesco et al. (2011) identificaram que mulheres com parto prematuro possuem 2,3 vezes mais chance de ter episiotomia, assim como o escore de Apgar no primeiro minuto de vida associase a episiotomia, indicando 2,1 vezes mais 
chance de sua ocorrência nos partos em que o recém-nascido nasce deprimido. As mulheres com bebês acima de $3.300 \mathrm{~g}$ têm 1,6 vezes mais chance de ocorrer laceração de segundo grau, o que pode justificar a indicação da episiotomia nesses casos, no entanto os autores recomendam a análise desta associação em pesquisas futuras, dada à divergência encontrada em outros estudos.

$\mathrm{Na}$ pesquisa realizada por Scarabotto e Riesco (2006), verificou-se que além do peso ao nascer, uma variável não apontada nos outros estudos: a presença de circular de cordão umbilical, cujos partos apresentam uma tendência à laceração mais profunda. $O$ peso do recém-nascido quando maior ou igual a $3.000 \mathrm{~g}$ apresentou um risco maior para o trauma perineal, no entanto, não foi evidenciado diferença estatisticamente significante com as outras faixas de peso. A vitalidade do recém-nascido apresentou-se preservada, com Apgar no primeiro e quinto minuto acima de 7 e nenhum bebê teve asfixia ao nascer. Esses achados mostram que a indicação da episiotomia como benefício para o recémnascido não é necessária.

\subsection{Condições assistenciais}

Na pesquisa de Mouta et al. (2008), os autores descreveram a frequência de episiotomia em partos na posição horizontal e vertical. Contudo, constataram que a posição materna no parto não se associa à utilização da episiotomia, sendo realizada, respectivamente em $28,9 \%$ e $29,1 \%$ dos casos.

Os estudos de Carvalho, Souza e Moraes Filho (2010) investigaram uma possível associação da realização de episiotomia com o turno de trabalho do profissional que as- siste a mulher no parto e com o uso de ocitocina e misoprostol, porém, os resultados não apresentaram significância estatística. Os autores inferem que a baixa frequência no uso do misoprostol pode estar relacionada a este resultado, enquanto que o uso indiscriminado da ocitocina dificulta a conclusão de resultados mais fidedignos. Contudo, verificou-se ainda que a frequência de trauma perineal espontâneo foi significativamente maior nas pacientes que não realizaram episiotomia. Em relação ao turno de trabalho do profissional, não foram encontradas evidências que fizessem essa associação.

Por outro lado, Melo Júnior, Lima e Freire (2006) constataram uma diferença significativa na realização da episiotomia conforme o turno de trabalho, sendo mais frequente no período diurno. Os autores acreditam que essa característica se deve ao fluxo intenso de parturientes durante esse período, levando o profissional a optar pela abreviação do tempo de trabalho de parto, a fim de atender a demanda.

Com relação à pesquisa de Costa e Souza (2009), foi analisado o tipo de profissional assistente no momento do parto em hospital público, onde identificou-se que a maioria era médico residente e a minoria enfermeiro residente e ambos apresentaram resultados semelhantes na realização da episiotomia, $45,5 \%$ e $50 \%$, respectivamente. Os autores ainda observam que os profissionais de enfermagem deveriam adotar condutas menos intervencionistas, indicando a urgente necessidade de revisar a formação desses profissionais e resgatar os conceitos de humanização no parto.

Nesse mesmo estudo, a episiotomia foi realizada em todos os partos em que foi usada a manobra de Kristeller associada à 
ocitocina e nos partos instrumentais, também associados à ocitocina.

Mouta et al. (2008) relacionaram a frequência de traumas perineais com a posição materna adotada durante o parto, no entanto, não houve diferença entre a posição vertical e a horizontal. Nesse contexto, em $63,2 \%$ dos partos realizados não houve episiotomia ou trauma perineal espontâneo.

Em estudo feito por Carvalho, Souza e Moraes Filho (2010) foram investigadas variáveis relacionadas à duração do trabalho de parto e do período expulsivo, porém não se observou associação destas com a realização da episiotomia, o que difere das recomendações do procedimento nos casos de trabalho de parto e período expulsivo prolongados.

A pesquisa realizada por Costa e Souza (2009) identificou utilização de episiotomia frequente no trabalho de parto com tempo médio de 6 horas; no entanto, resultados semelhantes foram observados também nos trabalhos de parto com o mesmo tempo de duração, porém, sem episiotomia. Scarabotto e Riesco (2006) também não identificaram associação significativa entre a duração do período expulsivo e a ocorrência de episiotomia.

\section{CONSIDERAÇÕES FINAIS}

Embora tenham sido encontrados poucos artigos na busca realizada, a revisão permitiu a identificação dos fatores relacionados com a episiotomia a partir das condições maternas, fetais e assistências e, além disso, levantaram-se questionamentos quanto a alguns critérios rotineiramente utilizados na adoção dessa prática. As muIheres primíparas e jovens são as mais sub- metidas à episiotomia; as condições fetais, embora abordadas como critério, apresentaram baixa associação à necessidade dessa prática; quanto às condições assistências, observou-se que os resultados não apresentam significância quanto à posição no parto, uso de ocitocina e misoprostol.

\section{REFERÊNCIAS}

AMORIN, M. M. R.; KATZ, L. O papel da episiotomia na obstetrícia moderna. Femina, v.36, n.1, p. 47-54, 2008.

BASTON, H.; HALL, J. 0 parto: uma abordagem humanizada. Rio de Janeiro: Elsevier; 2011.

CARROLLI, G.; MIGNINI, L. Episiotomy for vaginal birth (Cochrane Review). In: The Cochrane Library, Issue 1, 2012. Oxford: Update Software.

CARVALHO, C. C.; SOUZA, A. S. R.; MORAES FILHO, O. B. Episiotomia seletiva: avanços baseados em evidências. Femina, v.38, n.5, p. 266-270, 2010a. Prevalência e fatores associados à prática da episiotomia em maternidade escola do Recife, Pernambuco, Brasil. Revista Associação Média Brasileira, v. 56, n. 3, p. 333-339, 2010b.

COSTA, N. M. et al. Episiotomia nos partos normais: uma revisão de literatura. Facene/Famene, v. 9, n. 2, p. 45-50, 2011.

COSTA, L. C, SOUZA, L. M. Prevalência e correlação de fatores associados à prática de episiotomia em um hospital público do Distrito Federal. Comunicação em Ciências da Saúde, v. 20, n. 4, p. 314-324, 2009.

CUMIN, E. S.; FARIA, D. G. S.; SOLER, Z. A. S. G. Episiotomia de rotina versus episiotomia seletiva. Cuidarte, v. 1, n. 1, p. 100-3, 2007. 
GRAHAM, I. D. Episiotomy: challenging obstetric interventions. London: Blackwell Science; 1997. p. 33-41.

GUANILO, M. C. D. L. T. U.; TAKAHASHI, R. F. BERTOLOZZI, M. R. Revisão sistemática: noções gerais. Rev Esc Enferm USP, n. 45, v. 5, p. 1260-6, 2011.

KETLE, C. Anatomy of pelvic floor. In: HENDERSON, C.; BICK, D. editors. Perinned care: an international issue. London (UK): Quay Books, 2005.

LAKATOS, E. M. Fundamentos de metodologia científica. 6 ed. São Paulo: Atlas, 2005. LEAL, M. C.; et al. Obstetric interventions during labour and birth in Brazilian low risk women. Cad Saúde Public. v.30, n.4, p.1736, 2014.

MELO JÚNIOR, E. F.; LIMA, M. C., FREIRE, S. Fatores associados à realização seletiva de episiotomia em hospital universitário. Revista Ciências Médicas, v. 15, n. 2, p. 95101, 2006.

MOUTA, R. J. O et al. Relação entre posição adotada pela mulher no parto, integridade perineal e vitalidade do recém-nascido. Revista Enfermagem UERJ, Rio de Janeiro, v. 16, n. 4, p. 472-476, 2008.

OLIVEIRA, S. M. J. V, MIQUILINI, E. C. Frequência e critérios para indicar a episiotomia. Revista Escola de Enfermagem USP, v. 39, n.3, p. 288-295, 2005.

OMS, Organização Mundial de Saúde. Assistência ao Parto Normal: um guia prático. Saúde Materna e Neonatal/ Unidade de Maternidade Segura. Saúde reprodutiva e da Família. Genebra, 1996.

OULD, S. F. A treatise of midwifery. London: J Buckland; 1741.

PEREIRA, G. V.; PINTO, A. P. Episiotomia: uma revisão de literatura. Redalyc. Ensaios e Ciência. v. 15, n. 3, p. 184-196, 2011.

RIESCO, M. L. G, et al. Episiotomia, laceração e integridade perineal em partos normais: análise de fatores associados. Revista Enfermagem UERJ, Rio de Janeiro, v. 19, n. 1, p. 77-83, 2011.

SANTOS, A. R. dos. Metodologia científica: a construção do conhecimento. 5. ed. Rio de Janeiro: DP\&A, 2002.

SANTOS, J. O. et al. Frequencia de lesões perineais ocorridas nos partos vaginais em uma institução hospitalar. Escola Anna Nery Revista Enfermagem, Rio de Janeiro, v.12, n.4, p. 658-663, 2008.

SANTOS J. O; SHIMO, A. K. K. Discurso do sujeito coletivo das mulheres que sofreram episiotomia. Revista Mineira de Enfermagem, v.11, n.4, p. 432-438, 2007.

SCARABOTTO, L. B, RIESCO, M. L. G. Fatores relacionados ao trauma perineal no parto normal em nulíparas. Revista Escola Enfermagem USP, v. 40, n. 3, p. 389-395, 2006.

\section{(c) (1) \\ LYcense information: This is an open- access article distributed under the terms of the Creative Commons Attribution License, which permits unrestricted use, distribution, and reproduction in any medium, provi- ded the original work is properly cited.}

Artigo recebido em 10 de junho de 2015.

Avaliado em 26 de setembro de 2016.

Aceito em 24 de outubro de 2016.

Publicado em 03 de novembro de 2016.

\section{Como citar este artigo (ABNT):}

SANTOS, Rafael Cleison Silva dos; SANTOS, Rodrigo Gomes dos. Fatores relacionados com a prática da episiotomia no Brasil: revisão de literatura. Estação Científica (UNI- 
FAP), Macapá, v. 6, n. 2, p. 43-52, maio/ago.

2016. 